\title{
Holographic Equilibration of Nonrelativistic Plasmas
}

\author{
Umut Gürsoy, Aron Jansen, Watse Sybesma, and Stefan Vandoren \\ Institute for Theoretical Physics and Center for Extreme Matter and Emergent Phenomena, Utrecht University, \\ Leuvenlaan 4, 3584 CE Utrecht, The Netherlands \\ (Received 23 February 2016; revised manuscript received 25 May 2016; published 25 July 2016)
}

\begin{abstract}
We study far-from-equilibrium physics of strongly interacting plasmas at criticality and zero charge density for a wide range of dynamical scaling exponents $z$ in $d$ dimensions using holographic methods. In particular, we consider homogeneous isotropization of asymptotically Lifshitz black branes with full backreaction. We find stable evolution and equilibration times that exhibit small dependence of $z$ and are of the order of the inverse temperature. Performing a quasinormal mode analysis, we find a corresponding narrow range of relaxation times, fully characterized by the fraction $z /(d-1)$. For $z \geq d-1$, equilibration is overdamped, whereas for $z<d-1$, we find oscillatory behavior. Finally, and most interestingly, we observe that also the nonlinear evolution, although differing significantly from a quasinormal mode fit, is to a high degree of precision characterized by the fraction $z /(d-1)$.
\end{abstract}

DOI: 10.1103/PhysRevLett.117.051601

Introduction.-Quantum criticality has been a focus of interest both in theoretical and experimental physics over the past few decades. It is believed to be a key ingredient in the solution to the various yet unsolved problems such as the high $T_{c}$ superconductivity [1]. In particular, the dynamics of a system near a continuous quantum phase transition is governed by a universal, scale invariant theory characterized by the dimensionality $d$, the dynamical scaling exponent $z$, and the various other critical exponents that are independent of the microscopic Hamiltonian of the system. In these systems, the characteristic energy scale $\Delta$, such as the gap separating the first excited state from the ground state, vanishes as the correlation length $\xi$ diverges as $\Delta \sim \xi^{-z}$. For instance, $z=1$ occurs at the touching points of the band structure of monolayer graphene, $z=2$ can describe the case of bilayer graphene, and $z=2,3$ occur in heavy fermion systems; see, e.g., Refs. [2-6].

The existence of a quantum critical point at vanishing temperature determines the behavior of observables also at finite temperature, and even beyond the thermal equilibrium, in the so-called quantum critical region of the parameter space. In fact, a basic way to characterize this quantum critical region is to consider the response of the system to a small disturbance, determined by the equilibration time $\tau_{\text {eq }}$ [7]. The quantum critical region corresponds to short relaxation times $\tau_{\text {eq }} \sim 1 / T$ [8], whereas local equilibrium is reached much more slowly as $\tau_{\text {eq }} \gg 1 / T$ outside the quantum critical region [1].

In this Letter, we want to go one step further and ask the question, what happens when such a quantum critical system is taken completely out of equilibrium, when the perturbation is not small, but of the same order as the Hamiltonian? We answer this question partially in the particular situation when the collective excitations of the system are characterized by global, hydrodynamic quantities such as energy and pressure gradients at any time during the evolution. In this case, an example of such a large perturbation would be to consider a homogeneous, isotropic system with average pressure $P$ and create an initial anisotropy in one direction, say $\Delta P_{x}$, that is of the same order as $P$. The question then is how to characterize the evolution of this system towards equilibrium.

We will investigate equilibration processes in strongly interacting systems that can be modeled by holography [9-11]. There is a substantial amount of work in the literature that goes by the name holographic thermalization, concerning this problem in the case of relativistic scaling, $z=1$, following the seminal work of Ref. [12]. The problem of equilibration is mapped onto the evolution of a black brane geometry in the dual gravitational description. Thus, we obtain the fully nonlinear evolution of the black brane starting from the aforementioned initial conditions that correspond to anisotropy in pressure and we determine the isotropization of the system in time. In accordance with the earlier results for $z=1$, e.g., Refs. [13,14], we find that the system equilibrates quite rapidly, with isotropization times of the order $\sim 1 / T$. At later times, close to global thermal equilibrium, the evolution of the system is characterized by the quasinormal modes (QNMs) of the black brane [15]. In particular, the relaxation time $\tau$ above is related to the lowest lying quasinormal frequency as $\tau=-1 / \operatorname{Im} \omega_{0}$. We find that the relaxation times are determined by the ratio $z /(d-1)$ and fall in a narrow range. This agrees with our results of the nonlinear evolution.

Gravitational model.-The holographic description of field theories with Lifshitz scaling at criticality and zero temperature was initiated in Ref. [16]. To describe nonrelativistic plasmas holographically in the critical region at nonzero temperature, black brane solutions with Lifshitz asymptotics can be used [17]. The Hawking temperature 
of the black brane corresponds, via holography, to the temperature of the dual field theory. The action for this model is given by

$S=\frac{1}{16 \pi G} \int d^{d+1} x \sqrt{-g}\left[R-\Lambda-\frac{1}{2}(\partial \phi)^{2}-\frac{e^{\lambda \phi}}{4} F^{2}\right]$,

where $\Lambda=-(d+z-1)(d+z-2)$ is the cosmological constant, $\lambda=-\sqrt{2(d-1) /(z-1)}$, and $z$ is the dynamical scaling exponent, which is bounded by the null energy condition to be $z \geq 1$ [18]. The scalar field $\phi$ and the gauge field $F=d A$ are needed to support the Lifshitz geometry.

We consider an anisotropic but homogeneous system, which we describe using the following ansatz [19]:

$$
\begin{aligned}
d s^{2}= & -f(t, r) d t^{2}+2 r^{z-1} d t d r \\
& +S(t, r)^{2}\left[e^{(d-2) B(t, r)} d x_{1}^{2}+e^{-B(t, r)} d \vec{x}_{d-2}^{2}\right], \\
A= & a(r, t) d t, \quad \phi=\phi(r, t) .
\end{aligned}
$$

We take the gauge $A_{r}=0$, which is essential to obtain the nested form of the equations (6). The function $B(t, r)$ expresses the anisotropy of the black brane. The boundary, where the plasma lives, is at $r \rightarrow \infty$, and the horizon is denoted by $r_{H}$. From the viewpoint of holography this setup is dual to a nonrelativistic plasma with a pressure difference between the longitudinal direction $x_{1}$ and transversal directions $\vec{x}_{d-2}$.

By solving the equations of motion near the boundary, imposing Lifshitz asymptotics, we obtain

$$
\begin{aligned}
& f(r, t)=r^{2 z}+\mathcal{E} r^{z+1-d}-\frac{5}{8} \frac{\mathcal{P}(t)^{2}}{r^{2 d-2}}+\ldots, \\
& S(r, t)=r-n_{S} \frac{\mathcal{P}(t)^{2}}{r^{2(d+z)-3}}+\ldots, \\
& \phi(r, t)=\phi_{0} \log \frac{r^{2(d-1)}}{2(z-1)(z+d-1)}-\frac{2 n_{S} \mathcal{P}(t)^{2}}{r^{2(d+z-1)}}+\ldots,
\end{aligned}
$$

$a^{\prime}(r, t)=r^{z+d-2}-(d-1) n_{S} \frac{\mathcal{P}(t)^{2}}{r^{d+z}}+\ldots$,

$B(r, t)=\frac{\mathcal{P}(t)}{r^{d+z-1}}+\frac{1}{z} \frac{\partial_{t} \mathcal{P}(t)}{r^{d+2 z-1}}+\ldots$,

where $n_{S}=[(d-2) / 8](d+z-1) /[2(d-1)+z]$ and $\phi_{0}=$ $\sqrt{\frac{1}{2}(z-1) /(d-1)}$. In contrast to Ref. [12], we do not quench the system, but consider the equilibration of an out of equilibrium state, so we do not turn on a source for $B$.

In this expansion there are two free coefficients $\mathcal{E}$ and $\mathcal{P}$. The $\mathcal{E}$ is the normalizable mode of $f$ and it is proportional to the energy, which is required to be constant by the equations of motion. The function $\mathcal{P}$ is the normalizable mode of $B$ and will be related to the pressure difference. For $\mathcal{P}=0$ we recover the static black brane solution with $\mathcal{E}=-r_{H}^{d+z+1}$ [17]. The Hawking temperature is given by $T_{0}=[(d+z-1) / 4 \pi] r_{H}^{z}$. We stress that the scalar and gauge field do not have independent modes; they are completely determined by the metric and do not have any intrinsic dynamics.

To obtain vacuum expectation values, we need the counterterm action on the boundary. For our setup this can be obtained by generalizing, e.g., the analysis of Ref. [20] to arbitrary dimensions:

$S_{\mathrm{ct}}=\frac{1}{8 \pi G} \int d^{d} x \sqrt{-\gamma}\left[z-2 d-3+\frac{d+z-1}{2} e^{\lambda \phi} A^{2}\right]$.

Note that it breaks gauge invariance, but this is not an issue since the gauge field is not normalizable and not used to induce a chemical potential on the boundary [21]. For $z=1$, there is no gauge field, so the first term is enough.

Following Ref. [20], this yields a boundary energy momentum tensor in the coordinate basis $\left(t, x_{1}, \vec{x}_{d-2}\right)$,

$$
T_{\mu \nu}=\frac{N^{2}}{2 \pi^{2}} \operatorname{diag}\left(E, P_{L}, P_{T}, \ldots, P_{T}\right),
$$

where $E=-[(d-1) / 2] \mathcal{E}$ and $\Delta P \equiv P_{L}-P_{T}=[(d-1) \times$ $(d+z-1) / 2] \mathcal{P}(t)$. It satisfies the Ward identity $z E=P_{L}+(d-2) P_{T}$. In equilibrium, the pressure $P_{0}=-(z / 2) \mathcal{E}$. Furthermore, we abbreviated $N^{2} /\left(2 \pi^{2}\right)=$ $1 /(8 \pi G)$.

Numerical methods.-The numerical method we use to obtain solutions is an adaptation of Ref. [12] to asymptotically Lifshitz spacetimes. Using the ansatz in Eq. (2) and working with null derivatives $h^{\prime} \equiv \partial_{r} h$ and $\dot{h} \equiv \partial_{t} h+\frac{1}{2} r^{1-z} f \partial_{r} h$, the equations of motion can be put in a nested structure of linear ordinary differential equations (ODEs):

$$
\begin{aligned}
& 0=S^{\prime \prime}+\frac{1-z}{r} S^{\prime}+\frac{1}{2(d-1)} S \phi^{\prime 2}+\frac{d-2}{4} S B^{\prime 2}, \\
& 0=a^{\prime \prime}+a^{\prime}\left(\frac{1-z}{r}+(d-1) \frac{S^{\prime}}{S}+\lambda \phi^{\prime}\right), \\
& 0=(\dot{S})^{\prime}+(d-2) \frac{S^{\prime}}{S} \dot{S}+\frac{\Lambda r^{z-1} S}{2(d-1)}+\frac{r^{1-z} S e^{\lambda \phi}}{4(d-1)} a^{\prime 2}, \\
& 0=(\dot{B})^{\prime}+\frac{d-1}{2} \frac{S^{\prime}}{S} \dot{B}+\frac{d-1}{2} \frac{\dot{S}}{S} B^{\prime}, \\
& 0=(\dot{\phi})^{\prime}+\frac{d-1}{2} \frac{S^{\prime}}{S} \dot{\phi}+\frac{d-1}{2} \frac{\dot{S}}{S} \phi^{\prime}+\frac{\lambda}{4} r^{1-z} e^{\lambda \phi} a^{\prime 2},
\end{aligned}
$$



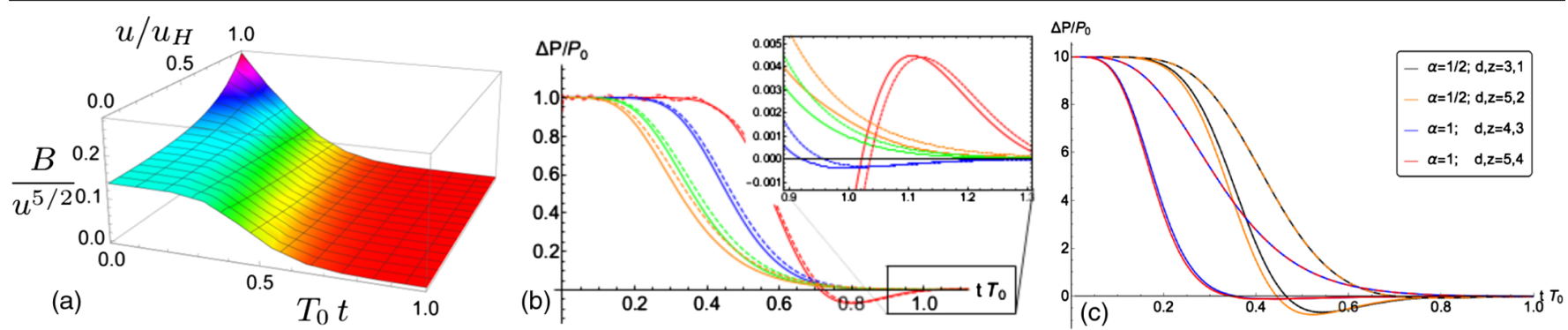

FIG. 1. (a) Nonlinear evolution of $B(u, t)$ for $d=4, z=2$. The colors indicate equal height. (b) Evolution of the pressure difference for $d=4$ and $z$ from 1 (rightmost) to 4 (leftmost). The inset shows a small oscillation below 0 for $z=2$. Dashed lines are the evolution resulting from a fit of the first 10 QNMs to the initial profile of $B$. (c) Evolution of pressure difference, comparing cases with the same $\alpha \equiv z /(d-1)$ but different $d$, showing close agreement. The initial profile is a factor of 10 bigger than in (b). Dashed lines denote evolution using the first 10 QNMs.

$$
\begin{aligned}
0= & f^{\prime \prime}+\frac{1-z}{r} f^{\prime}-2(d-1)(d-2) r^{z-1} \frac{\dot{S} S^{\prime}}{S^{2}}+r^{z-1} \dot{\phi} \phi^{\prime} \\
& +\frac{(d-1)(d-2)}{2} r^{z-1} \dot{B} B^{\prime}-\frac{d-3}{d-1} \Lambda r^{2 z-2} \\
& -\frac{13 d-5}{2} \frac{3 d-1}{d \phi} e^{\prime 2} \\
0= & \left(a^{i}\right)+a^{\prime}\left((d-1) \frac{\dot{S}}{S}+\lambda \dot{\phi}-\frac{z-1}{2} r^{-z} f\right) \\
0= & \ddot{S}+\frac{1}{2(d-1)} S \dot{\phi}^{2}-\frac{1}{2} r^{1-z} \dot{S} f^{\prime}+\frac{d-2}{4} S \dot{B}^{2} .
\end{aligned}
$$

Given initial profiles $B(t=0, r)$ and $\phi(t=0, r)$, the first equation is an ODE for $S$. Having solved this, the second becomes an ODE for $a^{\prime}$, and so we solve for the full geometry at $t=0$ by solving linear ODEs. In these equations we consider $\dot{S}$ independent of $S$, and similarly for the other functions. Numerically, we use pseudospectral methods [22] to solve these equations. All plots were generated with a grid of 40 points. After solving one time step, we can use $B, \dot{B}$, and $f$ to find $\partial_{t} B$ from the definition of the dot. We use a fourth-order Adams-Bashforth stepper to evolve this to the next time step. We do the same for $\phi$, and then start the procedure again [23].

For the numerics, we change the radial coordinate to $u=r^{-z}$. One computes the event horizon by solving the equation $\partial_{t} u_{H}(t) / u_{H}(t)^{2}=-(z / 2) f\left(u_{H}(t), t\right)$, arising from $d s^{2}=0$, with the boundary condition at late times $\left.f\left(u_{H}(t), t\right)\right|_{t \rightarrow \infty}=0$. We also have an apparent horizon, defined as the location of the largest trapped surface, given by $\dot{S}\left(u_{A H}(t), t\right)=0$. Using the Lifshitz scaling symmetry, we set $\mathcal{E}=-1$, so that at equilibrium $r_{H}=u_{H}=1$.

Results.-We analyze the nonlinear evolution for the cases $3 \leq d \leq 5$ and $1 \leq z \leq 4$. For the initial profiles we take the pressure to be $\Delta P(t=0) / P_{0}=1$. This defines $\mathcal{P}$ (we set time derivatives to 0 ), and to obtain consistent profiles we plug this into the near boundary expansion Eq. (3a).

We highlight the case $d=4$ and $z=2$, which is physically the most interesting. In Fig. 1(a) the evolution of the anisotropy function $B$ over the whole bulk spacetime is plotted. We checked that the entropy, as defined by the area density of either the event horizon or the apparent horizon, strictly increases during the evolution, as required by the area theorems. This is a consistency check of our numerics [24]. In all cases considered, the above consistency checks are valid.

The bulk evolution of $B$ describes the evolution of a pressure difference of the boundary theory through the function $\mathcal{P}$, which we turn to now. In Fig. 1(b) we show the time evolution of the pressure difference. The system isotropizes on a time scale of the order of the inverse temperature. Note first that for the cases where $z \geq d-1$, there are no oscillations, the system is overdamped. This will be more clear when we look at the QNMs, and is in agreement with [25].

The anisotropy $B$ obeys the massless Klein-Gordon equation at zero momentum. After redefining $\tilde{t}=z t$, this equation only depends on $d$ and $z$ through the ratio $\alpha \equiv z /(d-1)$, as

$\partial_{u}\left[u^{-1 / \alpha} \partial_{\tilde{t}} B-u^{2-1 / \alpha} f(u) \partial_{u} B\right]+u^{-1 / \alpha} \partial_{\tilde{t}} \partial_{u} B=0$,

with $f(u)=u^{-2}\left[1-\left(u / u_{H}\right)^{1+1 / \alpha}\right]$. Note that, in contrast, the nonlinear equations do depend on $d$ and $z$ separately. Furthermore, turning on either a mass or momentum for the QNM will also break the dependence on $\alpha$ alone. To compute QNMs we solve the associated generalized eigenvalue equation using pseudospectral methods [26].

We compare the nonlinear evolution with the sum of the first $10 \mathrm{QNMs}, B_{\mathrm{QNM}}(u, t)=\operatorname{Re} \sum_{i=0}^{9} c_{i} b_{i}(u) e^{-i \omega_{i} t}$. Here the $c_{i}$ are coefficients that we obtain by fitting to the initial profile $B(u, t=0), b_{i}$ are the eigenmodes, and $\omega_{i}$ the corresponding eigenfrequencies [27]. Dashed lines in Fig. 1(b) show the fitted QNM evolution. They always lag behind on the nonlinear evolution a little, but otherwise give a very good approximation. The late time evolution of the pressure is well approximated by the lowest QNM.

We check that taking different shapes as the initial profile, localized more in the UV or in the IR, gives a result similar to those in relativistic plasmas [in antide Sitter (AdS) space] [27], with profiles localized in the 


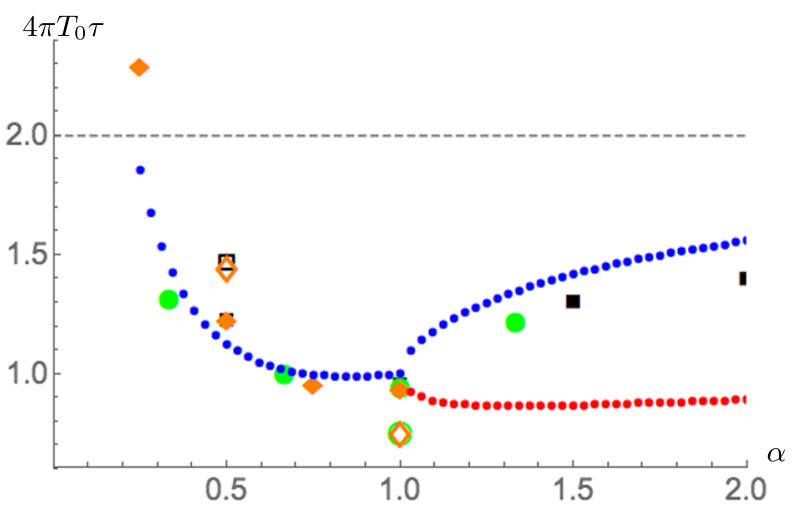

FIG. 2. Relaxation times from QNMs (blue points) as a function of $\alpha \equiv z /(d-1)$. At $\alpha=1$ the modes bifurcate, with the second lowest shown in red. Also shown are relaxation times $4 \pi T_{0} \tilde{\tau}$ obtained from the nonlinear evolution for $d=3$ (black squares), $d=4$ (green circles), and $d=5$ (orange diamonds). The hollow markers denote larger initial profiles.

UV better approximated by QNMs and those in the IR not well approximated.

For several cases of $(d, z)$ we were also able to study significantly larger profiles with $\Delta P(t=0) / P_{0}=10$, as shown in Fig. 1(c). Here we look at cases with the same value of $\alpha$ but different pairs of $(d, z)$. As expected, the fit with the first 10 QNMs is significantly worse than for the smaller profiles. More interestingly, though, the evolutions with the same $\alpha$ are very similar, their small difference being caused purely by the nonlinearities. Finally, we note that the larger profiles thermalize faster, as was already the case for AdS, but it is slightly more pronounced for higher $\alpha$.

Going back to the QNMs, for $\alpha \geq 1$ they become overdamped (purely imaginary) and we observe a bifurcation, see Fig. 2, with one mode branching upwards and the other downwards, converging as $\alpha \rightarrow \infty$ to the first two modes in $\mathrm{AdS}_{2}$. Note that $\alpha \rightarrow \infty$ can be interpreted as $z \rightarrow \infty$ at fixed $d$, or as $d \rightarrow 1$, at, e.g., $z=1$, matching Ref. [28] where it is stated that the $z \rightarrow \infty$ limit of Lifshitz corresponds to $\mathrm{AdS}_{2}$. This behavior is clarified in Fig. 3 where we plot the motion of the lowest QNMs in the complex plane, as we vary $\alpha$.

The value $\alpha=1$ can be found from $\mathrm{AdS}_{3}$, where one can show analytically that $\tau=1 /\left(4 \pi T_{0}\right)[25,29,30]$. Curiously, this is not the minimal value of the relaxation time, which instead sits at $\alpha \approx 0.847227$ and reads $\tau \approx$ $0.989002 /\left(4 \pi T_{0}\right)$. Note that the fact that the relaxation time increases again for larger $\alpha$ is also visible in the reversing of the green and orange lines in Fig. 1(c). For $\alpha=1+\epsilon^{2}$ with $\epsilon \ll 1$, we numerically find that $4 \pi T_{0} \tau \approx 1+\frac{1}{2} \epsilon$.

As $\alpha$ is taken to zero, the relaxation time diverges. However, we observe that with $d \leq 5$, which covers all physically relevant cases and implies that $\alpha \geq \frac{1}{4}$, we have a range of relaxation times $0.989002 \leq 4 \pi T_{0} \tau \leq 2$.

From the nonlinear evolution we obtain an isotropization time, defined as $\left|\Delta P\left(t \geq t_{\text {iso }}\right) / P_{0}\right| \leq \epsilon$, for some choice of $\epsilon$. To compare this with the relaxation time from the QNMs,

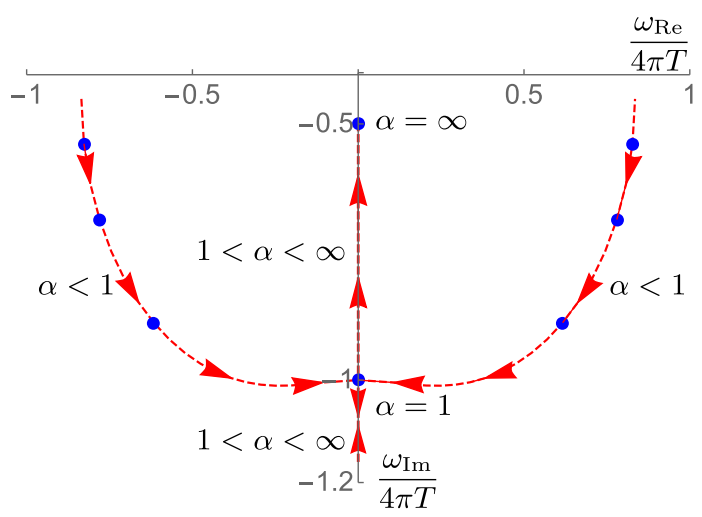

FIG. 3. We display the lowest modes found from Eq. (7), using numerics. Following the flow of the arrows corresponds to increasing $\alpha$. For $\alpha>1$, one mode moves up and asymptotes towards $\omega_{\operatorname{Im}} /(4 \pi T)=-0.5$ as $\alpha \rightarrow \infty$. The other mode moves down, initially, but at some point reverses and for $\alpha \rightarrow \infty$ asymptotes towards the $\alpha=1$ location. Dots denote points with $\alpha$ corresponding to an AdS space.

we define $\tilde{\tau} \equiv t_{\text {iso }} / \log \left[c_{0} b_{0}(0) / \epsilon\right]$. When applied to a single QNM decaying as $B_{0}(t)=c_{0} b_{0}(0) e^{-t / \tau}, \tilde{\tau}$ becomes equivalent to $\tau$.

Along with the relaxation times in Fig. 2, we plot $4 \pi T_{0} \tilde{\tau}$, with $c_{0}$ and $b_{0}(0)$ obtained from the QNM fit to the nonlinear evolution, taking $\epsilon=0.1$. We checked that choosing a smaller $\epsilon$ brings the nonlinear evolution closer to the evolution dictated by the QNMs, as expected, since smaller $\epsilon$ corresponds to later times. These have qualitatively the same dependence on $\alpha$, but generally lie just below $\tau$ due to the presence of higher order modes. As expected, the larger initial profiles deviate more from the QNM relaxation. There can be some noise due to oscillations, which are not accounted for in the definition of $\tilde{\tau}$, as seen in the point at $\alpha=1 / 4$ and $\alpha=1 / 2$.

Note also that at $\alpha=1$ we have the three cases $(d, z)=(3,2),(4,3)$, and $(5,4)$, which are very close to each other in the plot, and for $\alpha=1 / 2$ the cases $(d, z)=$ $(3,1)$ and $(5,2)$ nearly overlap, even for the large profiles.

Discussion.-We find that for physically relevant dimensions the relaxation time is roughly of the order $1 /\left(4 \pi T_{0}\right)$, with only mild $z$ dependence. We note that this universal behavior only applies to critical theories with no mass gap. Holographic equilibration in gapped theories such as QCD has also been studied in the literature [14,26,31], where the approach to equilibrium may be qualitatively different [14].

Our results are valid for large $N^{2} \sim 1 / G$ and strong coupling, but are consistent with the general arguments about equilibration times for general strongly coupled quantum critical systems (see, e.g., Ref. [1]). This seems to indicate that our results are quite robust and the large $N$ limit does not affect this behavior qualitatively.

We compared full nonlinear evolutions for different cases of $(d, z)$, which nevertheless have identical QNM spectra (the same $\alpha$ ), finding a remarkable agreement. So the fact that only the ratio $\alpha$ characterizes the evolution is 
even true to a high precision for the nonlinear evolution of large initial conditions. This stands in contrast with the fact that these evolutions are not approximated well by a QNM fit (the fit we perform with the first 10 modes has already converged; i.e., adding 10 more modes does not change the resulting evolution). Taken seriously, this suggests that isotropization in, e.g., heavy fermion systems $[5,6]$ in $d=4, z=3$ behaves similarly to isotropization in heavy fermion systems in $d=3, z=2$, up to finite order coupling corrections. It would be very interesting to perform a similar analysis in different Lifshitz models (see, e.g., Ref. [32]) to see if this is a common feature to all.

We thank Takaaki Ishii and Wilke van der Schee for comments on the draft. This work was supported by the Netherlands Organisation for Scientific Research (NWO) under VIDI Grant No. 680-47-518, VICI Grant No. 680-47-603, and the Delta-Institute for Theoretical Physics (D-ITP) that is funded by the Dutch Ministry of Education, Culture and Science (OCW).

[1] S. Sachdev, Quantum phase transitions, Wiley Online Library, (2007).

[2] S. A. Hartnoll, Lectures on holographic methods for condensed matter physics, Classical Quantum Gravity 26, 224002 (2009).

[3] A. H. C. Neto, F. Guinea, N. M. R. Peres, K. S. Novoselov, and A. K. Geim, The electronic properties of graphene, Rev. Mod. Phys. 81, 109 (2009).

[4] M. Killi, S. Wu, and A. Paramekanti, Band Structures of Bilayer Graphene Superlattices, Phys. Rev. Lett. 107, 086801 (2011).

[5] M. Garst, Aspects of Quantum Phase Transitions: Grüneisen Parameter, Dimensional Crossover and Coupled Impurities (Shaker Verlag, Herzogenrath/Maastricht, 2003).

[6] H. v. Löhneysen, A. Rosch, M. Vojta, and P. Wölfle, Fermiliquid instabilities at magnetic quantum phase transitions, Rev. Mod. Phys. 79, 1015 (2007).

[7] We use the terminology relaxation time for equilibration after small perturbations. Equilibration also occurs after large perturbations, and the isotropization we study in this Letter is an example of this. In that case, we speak of isotropization time.

[8] We work in units where $\hbar=k_{B}=c=1$.

[9] J. M. Maldacena, The Large $N$ limit of superconformal field theories and supergravity, Adv. Theor. Math. Phys. 2, 231 (1998).

[10] S. S. Gubser, I. R. Klebanov, and A. M. Polyakov, Gauge theory correlators from noncritical string theory, Phys. Lett. B 428, 105 (1998).

[11] E. Witten, Anti-de Sitter space and holography, Adv. Theor. Math. Phys. 2, 253 (1998).

[12] P. M. Chesler and L. G. Yaffe, Horizon Formation and Far-from-Equilibrium Isotropization in Supersymmetric Yang-Mills Plasma, Phys. Rev. Lett. 102, 211601 (2009).

[13] J. F. Fuini and L. G. Yaffe, Far-from-equilibrium dynamics of a strongly coupled non-Abelian plasma with non-zero charge density or external magnetic field, J. High Energy Phys. 07 (2015) 116.

[14] T. Ishii, E. Kiritsis, and C. Rosen, Thermalization in a holographic confining gauge theory, J. High Energy Phys. 08 (2015) 008.

[15] G. T. Horowitz and V. E. Hubeny, Quasinormal modes of AdS black holes and the approach to thermal equilibrium, Phys. Rev. D 62, 024027 (2000).

[16] S. Kachru, X. Liu, and M. Mulligan, Gravity duals of Lifshitz-like fixed points, Phys. Rev. D 78, 106005 (2008).

[17] M. Taylor, Non-relativistic holography, arXiv:0812.0530.

[18] C. Hoyos and P. Koroteev, On the null energy condition and causality in Lifshitz holography, Phys. Rev. D 82, 084002 (2010); Erratum: Null energy condition and causality in Lifshitz holography, Phys. Rev. D 82, 109905(E) (2010).

[19] Notice that this ansatz still has the gauge symmetry $r \rightarrow\left[r^{z}+\eta(t)\right]^{1 / z}$, which leaves the form invariant. In this Letter, $\eta=0$ is chosen.

[20] E. Kiritsis and Y. Matsuo, Charge-hyperscaling violating Lifshitz hydrodynamics from black-holes, J. High Energy Phys. 12 (2015) 076.

[21] Throughout this Letter we work at zero charge density. To introduce a chemical potential, one may use the black brane solution of Ref. [33].

[22] J. P. Boyd, Chebyshev and Fourier Spectral Methods (Dover Publications, Inc., Mineola, NY, 2001).

[23] There is a small subtlety here which does not arise in AdS. We have to specify initial profiles for both $B$ and $\phi$, but $\phi$ does not have any independent dynamics. Therefore, we must be careful to choose self-consistent initial profiles. We use the near boundary expansion to order $3(d+z-1)$ to achieve this.

[24] As a final check on the numerics, the constraint equation Eq. (6h) is small and decreases further with increasing precision.

[25] W. Sybesma and S. Vandoren, Lifshitz quasinormal modes and relaxation from holography, J. High Energy Phys. 05 (2015) 021.

[26] R. A. Janik, G. Plewa, H. Soltanpanahi, and M. Spalinski, Linearized nonequilibrium dynamics in nonconformal plasma, Phys. Rev. D 91, 126013 (2015).

[27] M. P. Heller, D. Mateos, W. van der Schee, and M. Triana, Holographic isotropization linearized, J. High Energy Phys. 09 (2013) 026.

[28] S. A. Hartnoll, J. Polchinski, E. Silverstein, and D. Tong, Towards strange metallic holography, J. High Energy Phys. 04 (2010) 120.

[29] D. Birmingham, I. Sachs, and S. N. Solodukhin, Conformal Field Theory Interpretation of Black Hole Quasinormal Modes, Phys. Rev. Lett. 88, 151301 (2002).

[30] Y.S. Myung and T. Moon, Quasinormal frequencies and thermodynamic quantities for the Lifshitz black holes, Phys. Rev. D 86, 024006 (2012).

[31] A. Buchel, M. P. Heller, and R. C. Myers, Equilibration Rates in a Strongly Coupled Nonconformal Quark-Gluon Plasma, Phys. Rev. Lett. 114, 251601 (2015).

[32] M. Taylor, Lifshitz holography, Classical Quantum Gravity 33, 033001 (2016).

[33] J. Tarrio and S. Vandoren, Black holes and black branes in Lifshitz spacetimes, J. High Energy Phys. 09 (2011) 017. 